Bereits bei Heranziehung von rund 20 Prozent der erfragten Zahlungsbereitschaft wird der absolute wirtschaftliche Vorteil eines Laufkraftwerkes durch die Wertschätzungen für die Natur aufgewogen, das heißt daß bei einer Zahlungsbereitschaft von 64 Schilling pro Jahr und ÖsterreicherIn (über 14 Jahre) der Barwert der Variante 2 (Nationalpark-Maximalvariante) größer ist als der Barwert der „,besten“ Variante mit Kraftwerk.

Dies zeigt auch die Abbildung 1, in der die Barwerte der volkswirtschaftlichen Kosten-NutzenSaldi der verschiedenen Gestaltungsvarianten des österreichischen Donau-Abschnitts dargestellt sind. Die Barwerte wurden in Abhängigkeit von der mittleren jährlichen Zahlungsbereitschaft (in Schilling) der Österreicher über 14 Jahre für Augebiete errechnet. Dabei wurde ein realer Diskontierungszinssatz von zwei Prozent pro Jahr (von 1993 bis 2086) zugrunde gelegt.

Es zeigt sich, daß je höher die Zahlungsbereitschaft für einen Nationalpark ist, desto größer ist der Barwert des Nationalparks und desto geringer jener der größten Variante mit Kraftwerk (mit steigender Zahlungsbereitschaft für Augebiete entlang freier Fließstrecke nehmen die geldmäßig bewerteten Verluste durch ein Lauftkraftwerk $\mathrm{zu}$; damit sinkt der Barwert der Variante 3.2).

\section{Die Bedeutung der Kosten-Nutzen-Analyse}

Zusammenfassend läßt sich festhalten, daß die Kosten-Nutzen-Analyse (KNA) auswählter Varianten eines Nationalparks Donau-Auen folgende Zielsetzungen erfïllte:

- Die KNA diente zur Informationsbeschaffung für die EntscheidunsträgerInnen und Betroffenen, da erstmals aus wirtschaftswissenschaftlicher Sicht die einzelnen Problemdimensionen in ihrer volkswirtschaftlichen Bedeutung beurteilt werden konnten.

- Die KNA machte weiter jene kritischen Projektbestandteile transparent, die je nach Ausprägung zu gänzlich unterschiedlichen Ergebnissen führte.

- Die Messung der volkswirtschaftlichen Effizienz eines Projektes mittels der KNA mag ein abstrakter Wunsch der Ökonomie als Wissenschaft sein, jedoch sagt der volkswirtschaftliche, Vermögenszuwachs aufgrund der Durchführung eines Projektes noch sehr wenig über die Erwünschtheit eines Projektes bei einer Vielzahl weitergehender Anforderungen (Verteilungsprobleme, Akzeptanz der Anrainergemeinden usw.) aus.

- Eine ökologisch sensible und auf erfragte Zahlungsbereitschaften zurückgreifende KNA kann sich im Zusammenhang mit der Bewertung öffentlicher Projekte als umweltpolitisches Instrument erweisen, da die Präferenzen der Bevölkerung für den Erhalt oder die Verbesserung von Naturgütern auch geldmäßiges Gewicht erhält.

- Das Instrument der Zahlungsbereitschaftsbefragung selbst ist jedoch kaum allgemein vermittelbar, und die Frage, was geäußerte Zahlungsbereitschaften wirklich aussagen, größtenteils noch immer ungelöst.

\section{Anmerkungen}

1) Da aufgrund der röumlichen Struktur des Gebietes und der Vielzahl an Eintrittsmöglichkeiten kein Eintrittspreis erhoben wird und ouch politisch ein Eintrittspreis nicht durchsetzbar und wünschbar ist, handelt es sich lediglich um die Zohlungsbereitschaft für einen hypothetischen Eintrittspreis.
2) Dies ist jedenfalls ein wichtiges Ziel der Kosten-NutzenAnalyse. An dieser Stelle kann jedoch nicht darauf eingegangen werden, ob die zugrundeliegende Annahme der Substituierbarkeit zwischen Naturgütern und "man-made capitol" tatsächlich im unterstellten Umfang gegeben ist, und inwiefern die Kosten-Nutzen-Analyse überhaupt ein geeignetes Instrument zur Beurteilung öffentlicher Projekte darstellt.

\section{Literafur}

- M. Kosz: Valuing Riverside Wetlands: The Case of the "Donau-Auen" National Park. Ecological Economics 16 (2) 1996, 109-127.

- W. Schönbäck., M. Kosz, T. Madreiter, D. Gfall, B. Brezino: Kosten-Nutzen-Analyse ausgewählter Vorianten eines Nationalparks Donau-Auen. Springer Verlag, Wien - New York (erscheint im Herbst 1996).

\section{Der Autor}

Dr. Michael Kosz ist wissenschaftlicher Mitarbeiter am Instifut für Finanzwissenschaft und Infrastrukturpolitik

Kontakt: Technische Universität Wien, 1040 Wien, Karlsgasse 11, Tel. $(++43)$ (1) $58801-4321$, Fax 50427 38, E-Mail:mkosz@email.tuwien.ac.at

\title{
Von der City-Logistik zur raumbezogenen Stadtlogistik
}

\section{City-Logistik ist das akfuelle Schlagwort im städtischen Güterverkehr. Damit werden große Hoffnungen hinsichtlich Transportoptimierung (öberwiegend) und Umweltentlastung (teilweise) verbunden. Seit einigen Jahren werden darunter verstärkt organisatorische Innovationen für eine Neugestalfung der städtischen Warentransporte diskutiert und praktisch erprobt - so auch in den hier vorge- stellten Modellvorhaben.}

$\mathrm{E}$ Von Markus Hesse und Silke Kruspe nde 1995 wurde bereits in rund 80 deutschen Städten über den städtischen Güterund Lieferverkehr diskutiert und in 19 Städten sind nach Angaben des Bundesverbandes Logistik (BVL) e.V. bereits konkrete Aktivitäten und Projekte in Gang gesetzt worden. Diese Aktivitäten bestehen überwiegend aus Kooperationen und Pools zur Belieferung von Problemkunden und -zonen durch Speditionen (vgl. Logistik Heute 1995). Ausgehend von einigen Anfangserfolgen in der Initiierung und Erprobung von
Speditionskooperationen - teilweise rein privat, teilweise unter Beteiligung der öffentlichen Hand - wurden mit dem Schlagwort der „CityLogistik“ hohe Erwartungen geweckt. Doch konnte die praktische Umsetzung dieser Modelle vor dem Hintergrund verschiedener, zum Teil nicht deckungsgleicher Ziele, diese bisher noch nicht erfüllen. Die Erfahrungen zeigen zwar, daß entgegen anfänglicher Erwartungen Kooperation auch im Wettbewerb möglich ist. Aus Sicht der Planung mangelt es jedoch an einem überzeugenden Entwurf für eine funktionstïch- 
tige Gestaltung des gesamten Wirtschaftsverkehrs in der Stadt. Eine grundlegende Notwendigkeit besteht daher darin, Umsetzungserfahrungen zu sammeln und den Wirkungsrahmen einer erfolgreich initiierten City-Logistik zu erweitern.

Damit sind nachvollziehbare und spürbare Effekte im Stadtraum gemeint (Verkehrsreduzierung, Abbau des Störpotentials durch Schwerverkehre, Städtebau), die über die Optimierung einzelner Teilsegmente wie die speditionelle Distribution deutlich hinausgehen. Im Mittelpunkt einer solchen Strategie steht der Übergang von der punktuell agierenden City-Logistik zur raumbezogenen Stadtlogistik.

\section{Modellvorhaben in Nordrhein- Westfalen}

Das Ministerium für Stadtentwicklung, Kultur und Sport des Landes Nordrhein-Westfalen hat vor diesem Hintergrund im Jahre 1995 die exemplarische Förderung von Modellprojekten einer erweiterten Stadtlogistik ausgeschrieben. Aus den Bewerbungen der Städte und Gemeinden in NRW wurden 20 Modellvorhaben bzw. Bausteine ausgewählt, die in den kommenden fünf Jahren als public-private-partnership durchgeführt werden sollen und mit insgesamt 50 Millionen Mark durch das Land gefördert werden.

Die Durchführung dieser Modellvorhaben dient im wesentlichen folgenden Zielen: Erstens soll generell der Kenntnisstand in der konkreten Umsetzung von City- und Stadtlogistik verbessert und erweitert werden, vor allem bezüglich der Handlungsbedingungen und Handlungsmöglichkeiten örtlicher Akteure (Abbau von Praxisdefiziten, Sammlung von Implementationserfahrungen). Zweitens soll es zu einer Erweiterung der vorliegenden Ansätze und Aktivitäten zur CityLogistik kommen, insbesondere bezogen auf:

- unterschiedliche Akteure und Branchen (nicht nur Transportwirtschaft, sondern auch Verlader und Handel bzw. EndverbraucherHaushalte),

- unterschiedliche Teilräume (nicht nur Innenstädte, sondern auch Nebenzentren, denkmalgeschützte Bereiche, Gewerbegebiete usw.),

- verschiedene Verkehrsträger (Verkehrsverlagerung durch Einsatz von ÖPNV, Fahrrädern oder Leichtfahrzeugen im Nahverkehr bzw. der Bahn im Fernverkehr) sowie

- verschiedene Ziele( es gilt nicht nur Verkehrsoptimierungen aus funktionaler Sicht zu suchen,sondern auch Lösungsansätze die aus städtebaulich-ökologischer Sicht sinnvoll erscheinen).

Drittens sollen Defizite im praktischen Handling des notwendigen Kommunikationsprozesses beseitigt werden, vor allem durch den innovativen Umgang mit public-private-partnership. Damit ist beabsichtigt, die Effizienz der Planung durch eine zielgerichtete, konfliktfähige Kommunikation und Kooperation zwischen Unternehmen und öffentlicher Hand zu verbessern.

\section{Kommunale Aktivitäten}

Unter den ausgewählten Modellvorhaben ist ein sehr breites Spektrum von Stadtstrukturen, Problemen und Handlungsstrategien vertreten. Thematisch richten sich die Modellvorhaben nicht nur auf die bereits vielfach diskutierten Ansätze in den Bereichen Spedition bzw. Handelsdistribution oder Straße-Schiene-Terminals, sondern auch auf den Werkverkehr, andere Branchen (z.B. Baugewerbe, Entsorgung), andere Verkehrsträger (Bahn, Binnenschiff, Fahrrad) oder die Einbeziehung von Zustelldiensten für private Haushalte. Acht der ausgewählten Vorhaben sind in den Zentren der großen Ballungsräume lokalisiert (überwiegend Rhein-Ruhr). Weitere Projekte vertreten Siedlungstypen wie die Ballungsrandzone, zwei solitäre Verdichtungsgebiete und sechs Kommunen im ländlichen Raum.

Viele Projekte befinden sich erst in der Konzeptionierungsphase und beginnen gerade mit einer Problematisierung der Stadtlogistik. Acht der vorgeschlagenen Modellvorhaben besitzen schon einen Vorlauf in der Bearbeitung des Themas Güter- und Wirtschaftsverkehr, dort werden bereits Aktivitäten zur Bündelung von Lieferverkehr, zur Verbesserung des Schienenverkehrs usw. unternommen, auf die das jeweilige Modellvorhaben aufbauen kann. Einen besonderen Stellenwert besitzen die Probleme von Klein- und Mittelstädten, in denen bisher relativ wenig Erfahrungen in der Thematisierung und Behandlung von Problemen der Stadtlogistik gemacht wurden und in denen auch der Problemdruck objektiv und subjektiv ein anderer ist als in den Kernbereichen der großen Städte.

\section{- Wissenschaftliche Begleit- forschung}

Eine wissenschaftliche Begleitforschung wird das Land Nordrhein-Westfalen über die Laufzeit der Modellvorhaben unterstïtzen. Ziel ist es, Wirkungsweisen, Erfolge und Hemmnisse der
Stadtlogistik in der Praxis transparent zu machen. Die Begleitforschung wird kontinuierlich über den jeweiligen Stand der Ergebnisse berichten. Zur projektïbergreifenden Kommunikation wird zur Zeit ein Informationsnetzwerk Stadtlogistik NRW errichtet. Dieses Netzwerk soll in erster Linie den Erfahrungsaustausch unter den Beteiligten in den Modellstädten und -gemeinden fördern. Im Rahmen dieser Art interkommunaler Kooperation können Synergieeffekte besser genutzt und das im Modellvorhaben erworbene Wissen gebündelt werden. Auch wird es einen gemeinsamen Arbeitskreis geben, die sogenannte Plattform, wo inhaltliche und organisatorische Fragen und Probleme gemeinsam zwischen den Modellgemeinden, dem zuständigen Ministerium und der Begleitforschung diskutiert werden. Ferner sollen innerhalb des Netzwerkes Arbeitsgruppen zu den unterschiedlichen methodischen Ansätzen zur Stadtlogistik eingerichtet werden, in denen an spezifischen Fragestellungen und Lösungsansätzen gearbeitet werden soll.

Für den Austausch und die Kommunikation innerhalb (und außerhalb) des Netzwerks wird das FORUM Stadtlogistik NRW eingerichtet: Es handelt sich dabei um einen Informationsdienst, durch den alle Beteiligten auf informellem Wege miteinander kommunizieren können und über das Modellvorhaben informiert werden. Außerdem sollen so auch außenstehende Interessentèn über die Modellvorhaben informiert werden.

\section{Ziele und Wirkungen}

Ein wichtiges Ziel der Begleitforschung und kritischer Punkt vieler Konzepte und Einzelmaßnahmen ist die Frage nach den durch Stadtlogistik tatsächlich erreichbaren Entlastungen und (vor allem stadtraumbezogenen) Wirkungen. Gemessen an den je nach Interessenlage zudem sehr unterschiedlichen Anforderungen an die Theorie der Stadtlogistik dürfte es unbefriedigend sein, daß die bisher aus ihrer Praxis resultierenden Effekte nur sehr partiell Wirkungen entfalten. Zudem sind sie nur schwer meßbar beziehungsweise überprüfbar, da sie in aller Regel auf bestimmte Teilsegmente des Wirtschaftsverkehrs beschränkt sind. Der Atomisierung der Sendungsgrößen und Güterströme entspricht eine extreme Zersplitterung der logistischen Kanäle und Handlungsfelder.

Neben den allgemeinen Wirkungen von Maßnahmen auf die Verkehrsmenge sollten daher auch die städtebaulichen Entlastungseffekte 
punktueller Maßnahmen beachtet werden, die sich positiv auf die städtische Umwelt auswirken könnten. Mittelfristig entscheidend ist unseres Erachtens aber nicht das n-te Prozent Belastungsminderung, sondern einzelne Schritte bei der Entwicklung perspektivischer Vorstellungen über den Wirtschaftsverkehr, um Wirtschaft und Verkehr wieder ein Stiuck in die Stadt zu reintegrieren. Hinzu kommt eine nicht unwesentliche Wirkung auf die Diskussion über den gesamten Stadtverkehr. Gelingt eine praxistaugliche Regulierung des Wirtschaftsverkehrs vor Ort, so bestehen auch Handlungsspielräume und weniger Konfliktpotential in bezug auf die Reduzierung des privaten Pkw-Verkehrs, denn bekanntlich werden der Wirtschaftsverkehr und der Personenverkehr nicht selten gegeneinander ausgespielt: Statt dessen sollten das Problembewußtsein und die Handlungskompetenzen der Stadtpolitik allgemein gestärkt werden. Wesentlich erscheint uns dazu, daß auf der Seite der öffentlichen Sache aktive, selbstbewußte Akteure stehen, die ihre Verpflichtung zur Abwägung unterschiedlicher Interessen wahrnehmen, eigene Ziele einbringen und ihren Auftrag nicht allein in der Moderation der Interessen externer Anspruchsgruppen sehen.

\section{Literafur}

- H. Boes, M. Hesse: Güterverkehr in der Region. Technik, Organisation, Innovation. Metropolis-Verlag, Marburg 1996

- Logistik-Heute: Sonderausgabe "City-Logistik", 10/1995

- Ministerium für Stadtentwicklung, Kultur und Sport des Londes Nordrhein-Westfalen: Auftaktkongreß "Stadtlogistik na logo!" am 12. Dezember 1995 in Gütersloh. Redemanuskripte, Düsseldorf

\section{Die Autoren}

Markus Hesse betreut das Forschungsfeld "Stadtentwicklung, Planung, Verkehr" des IÖW in Berlin, Tel. (030) 88459424

Silke Kruspe ist als Diplom-Geographin in diesem Forschungsfeld tätig, lÖW-Regionalbüro NRW, Wup pertal, Tel. (0202) 80530

\section{Meldungen}

\section{Regionale Wirtschaftskreișläufe}

Seit Anfang des Jahres werden in den neuen Bundesländern fünf Projekte zur Sicherung und zum Wiederaufbau regionaler Wirtschaftskreisläufe durchgeführt. Die ersten Erfolge der von der Deutschen Bundesstiftung Umwelt geförderten Projekte wurden Ende Juli im Mansfelder Land bei Halle/Saale präsentiert. Gemeinsame Grundidee aller Projekte ist, daß Naturschutz heute als Querschnittsaufgabe begriffen werden muß, die den Menschen nicht aussperrt, sondern ihn in allen seinen Tätigkeiten als Handelnden in die Konzepte mit einbezieht.

Noch vor zehn Jahren, als die ersten Landschaftspflegeverbände in Deutschland gegründet wurden, war ihr Hauptanliegen, wertvolle Biotopflächen $\mathrm{zu}$ erhalten und Landwirten ein Zusatzeinkommen in der Landespflege zu sichern. Sowohl das Selbstverständnis als auch das Tätigkeitsfeld der heute 120 Verbände hat sich in den letzten Jahren aber beträchtlich verändert. Dies ist auf die Einsicht zurückzuführen, daß vielfältige Kulturlandschaften nur zu erhalten sind, wenn die dort umweltschonend erzeugten Produkte auch einen Markt finden und damit die Wirtschaftskraft der ländlichen Räume gestärkt wird. Der Aufbau regionaler Wirtschaftskreisläufe ist damit ein wichtiger Beitrag zur umweltgerechten Landnutzung.

Kontakt: Deutscher Verband für Landschaftspflege,

Eyber Str. 2, 91522 Ansbach, Tel. (0981) 9504247

\section{Umwelt- und Güterverkehrs- politik}

Inwieweit wird Umweltschutz in der Güterverkehrspolitik der Europäischen Union berücksichtigt? Wie ist die Situation in diesem Politikfeld $\mathrm{zu}$ bewerten, zu erklären und $\mathrm{zu}$ verbessern? Anworten darauf wurden in einem Forschungsprojekt am Institut für regionale Studien in Europa (EURES) erarbeitet. Die sechs in diesem Projekt verglichenen Länder - Italien, Schweiz, Deutschland, Niederlande, Dänemark und Großbritannien - repräsentieren nicht nur unterschiedliche ökonomische Strukturen, politische Systeme etc., sondern auch unterschiedliche verkehrspolitische Ordnungsvorstellungen. Gemeinsam ist ihnen aber eine Verkehrspolitik, die durch den Vorrang wachstumsorientierter und anderer wirtschaftlicher Ziele bestimmt ist.

Es gibt (noch) kein Musterland ökologischer Güterverkehrspolitik. Aber es existieren immerhin interessante Detailerfahrungen: die Wirksamkeit ökologischer Ziele für den Verkehr und die Praxis einer strategischen Umweltverträglichkeitsprüfung in den Niederlanden und Dänemark, die klare Bevorzugung der Schiene in der Alpentransitpolitik der Schweiz, die deutsche
Bahnreform oder die britische Vorreiterrolle bei der Besteuerung des Verkehrs.

Insgesamt kamen die Projektbearbeiter zu dem Schluß, daß die Rechtsposition der Umweltpolitik aber gegenüber der Verkehrspolitik in allen Ländern relativ schwach ist. Schuld daran sei die häufige Vermeidung klarer sektoraler Umweltziele. Außerdem sei das Querschnittsprinzip der Umweltpolitik bisher unzureichend definiert. Die Projektergebnisse sind zum Teil als EURES discussion paper veröffentlicht.

Kontakt: EURES, Baslestr. 19, 79100 Freiburg, Fax (0761) 7044144

\section{Öko-Audit an der Fachhochschule}

Die Fachhochschule für Wirtschaft (FHW) in Berlin kann erste Ergebnisse und Ziele ihres Projekts "Öko-Audit der Fachhochschule“ bekannt geben. Bereits 1995 fiel der Startschuß, als im Rahmen einer Diplomarbeit die grundsätzliche Übertragbarkeit der EMAS-Verordnung auf den Dienstleistungssektor untersucht wurde. Mit Hilfe einer eigens dafür entwickelten Checkliste wurde dann die erste Umweltprüfung der Fachhochschule durchgefuihrt und ein umfassender Prüfbericht erstellt. Im Mai dieses Jahres beschloß das Kuratorium der FHW schließlich sogar umweltpolitische Leitlinien, die in Zusammenarbeit mit verschiedenen Mitgliedern der Hochschule entwickelt wurden. Sie beschreiben die strategischen Gesamtziele und operativen Handlungsgrundsätze, die auch in Zukunft die Berücksichtigung des Faktors Umwelt bei allen Tätigkeiten, Planungen und Entscheidungen an der FHW sicherstellen.

Als „Umweltgutachter“ fungieren an der FHW Studierende des vier Semester umfassenden Aufbaustudiengangs „Umweltmanagement“. Ziel des Projektes ist es, neben der eigenen Auditierung, theoretische Grundlagen und Zielsetzungen für künftige Öko-Auditierungen im Dienstleistungssektor zu erarbeiten. Dazu kann die modellhafte Umsetzung an der FHW direkt genutzt werden. Denn die Öko-Audit- bzw. EMAS-Verordnung spricht zur Zeit zwar nur Unternehmen des produzierenden Sektors an, es kann aber mit einer Ausweitung auf den Dienstleistungssektor gerechnet werden.

Am 15. November 1996 wird für Interessierte an der FHW ein Werkstattgespräch zum Thema Öko-Audit an Hochschulen - Erfahrungen und Perspektiven stattfinden. Hierzu werden auch 
(c) 20I0 Authors; licensee IÖW and oekom verlag. This is an article distributed under the terms of the Creative Commons Attribution Non-Commercial No Derivates License (http://creativecommons.org/licenses/by-nc-nd/3.o/), which permits unrestricted use, distribution, and reproduction in any medium, provided the original work is properly cited. 